\section{Infected Developmental Cyst of the Esophagus Mimicking Stromal Cell Tumor}

A 38-year-old man was admitted to the hospital because of low grade fever and atypical chest pain. Barium swallow findings were consistent with a submucosal tumor in the distal esophagus and endoscopic examination showed a submucosal lesion without mucosal abnormalities close to the esophagogastric junction.

Endoscopic ultrasonography (EUS) (GFUM 20; Olympus, Tokyo, Japan) demonstrated a mass contiguous with the fourth hypoechoic layer (muscularis propria) of the esophagus, with an approximate size of $3.8 \times 2.3 \mathrm{~cm}$ and an hypoechoic center which was interpretated as a necrotic area (Figure 1). Several round lymph nodes greater than $1 \mathrm{~cm}$ were also seen adjacent to the lesion. The findings were strongly suggestive of a stromal cell tumor.

The size of the lesion, and the presence of a central necrotic area and surrounding lymph nodes suggesting malignancy, led to a decision for surgical resection. The patient underwent an esophagectomy and a proximal partial gastrectomy with regional lymph node dissection, without complications, and he remained free of symptoms 12 months later.

Macroscopically, the lesion was an homogeneous soft tissue tumor, with a nodular and irregular rim of peritumoral fat tissue, contiguous with the esophagus. The histological examination showed a cystic lesion open to the esophageal lumen, lined by respiratory epithelium and surrounded by a layer of smooth muscle, which was diagnostic of developmental cyst (Figure 2).

Most large developmental cysts are considered to be duplication anomalies, composed of a central mucosa-lined lumen surrounded by thick layers of smooth muscle which could be continuous with the muscularis propria, or even within it [1]. They may resemble submucosal tumors endoscopically [2], but EUS usually shows a cystic lesion leading to the diagnosis of esophageal cyst (bronchogenic or developmental cyst, or cystic dilation of esophageal glands). In this case the infection of the cyst caused significant inflammatory changes, with wall thickening and the presence of lymph nodes giving the endosonographic appearance of a degenerated stromal tumor [3].

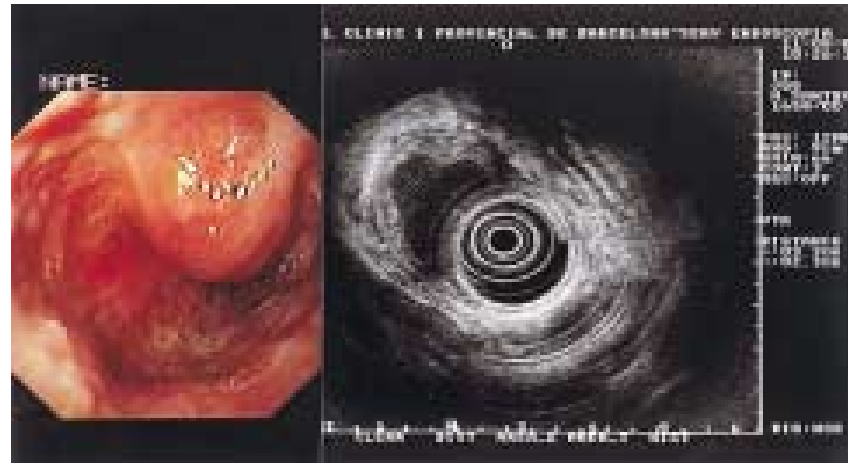

Figure 1 Endoscopic (left) and endoscopic ultrasound (EUS) (right) appearance of the lesion, mimicking a submucosal tumor with an hypoechogenic center interpreted as a necrotic area on EUS

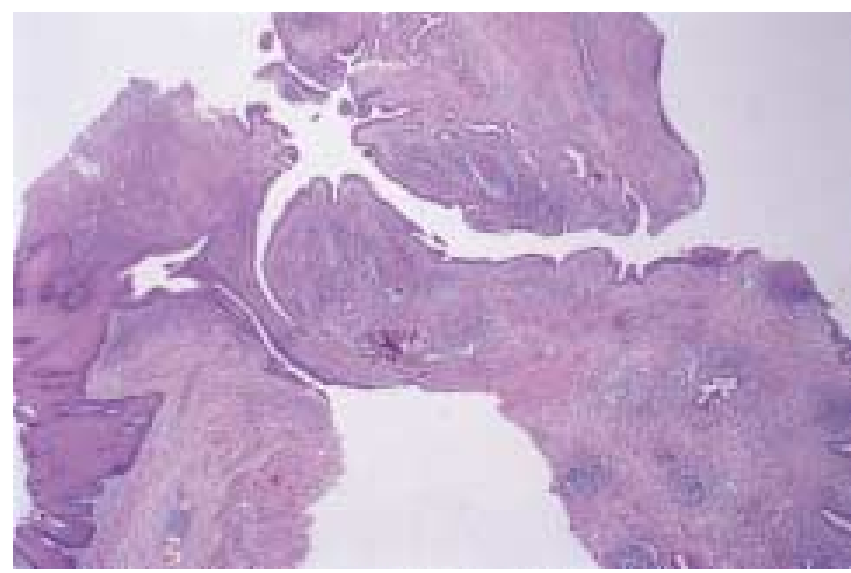

Figure 2 Histological examination showed normal squamous esophageal epithelium (on the right of the figure) and a fistulous tract which opened to the cyst. The cyst wall was lined by respiratory epithelium

M. T. Soria ${ }^{1}$, A. Ginès $^{1}$, I. Blesa ${ }^{1}$, J. Llach ${ }^{1}$, G. Fernández-Esparrach ${ }^{1}$, M. Pellisé ${ }^{1}$, L. Argüello $^{1}$, R. Miquel $^{2}$, M. Pera ${ }^{3}, J$. M. Bordas ${ }^{1}$

${ }^{1}$ Endoscopy Unit, Institut de Malalties

Digestives, Hospital Clínic,

University of Barcelona, Spain

${ }^{2}$ Dept. of Pathology, Institut de Malalties

Digestives, Hospital Clínic,

University of Barcelona, Spain

${ }^{3}$ Dept. of Surgery, Institut de Malalties

Digestives, Hospital Clínic,

University of Barcelona, Spain

\section{References}

${ }^{1}$ Geller A, Wang KK, DiMagno EP. Diagnosis of foregut duplication cysts by endoscopic ultrasonography. Gastroenterology 1995; 109: 838-842
${ }^{2}$ Yasuda K, Cho E, Nakajima M, et al. Diagnosis of submucosal lesions of the upper gastrointestinal tract by endoscopic ultrasonography. Gastrointest Endosc 1990; 36: S17-S20

${ }^{3}$ Chak A, Canto I, Rösch T, et al. Endosonographic differentation of benign and malignant stromal cell tumors. Gastrointest Endosc 1997; 45: 468-473

Corresponding Author

A. Ginès, M.D.

Endoscopy Unit

Institut de Malalties Digestives

Hospital Clínic

Villaroel 170

08036 Barcelona

Spain

Fax: $\quad+34-93-2279387$

E-mail: magines@medicina.ub.es 